Document downloaded from:

http://hdl.handle.net/10251/49160

This paper must be cited as:

Gomis Hilario, O.; Vilaplana Cerda, RI.; Manjón, F.; Santamaría Pérez, D.; Errandonea, D.; Pérez González, E.; López Solano, J.... (2013). Crystal structure of HgGa2Se4 under compression. Materials Research Bulletin. 48:2128-2133. doi:10.1016/j.materresbull.2013.02.037.

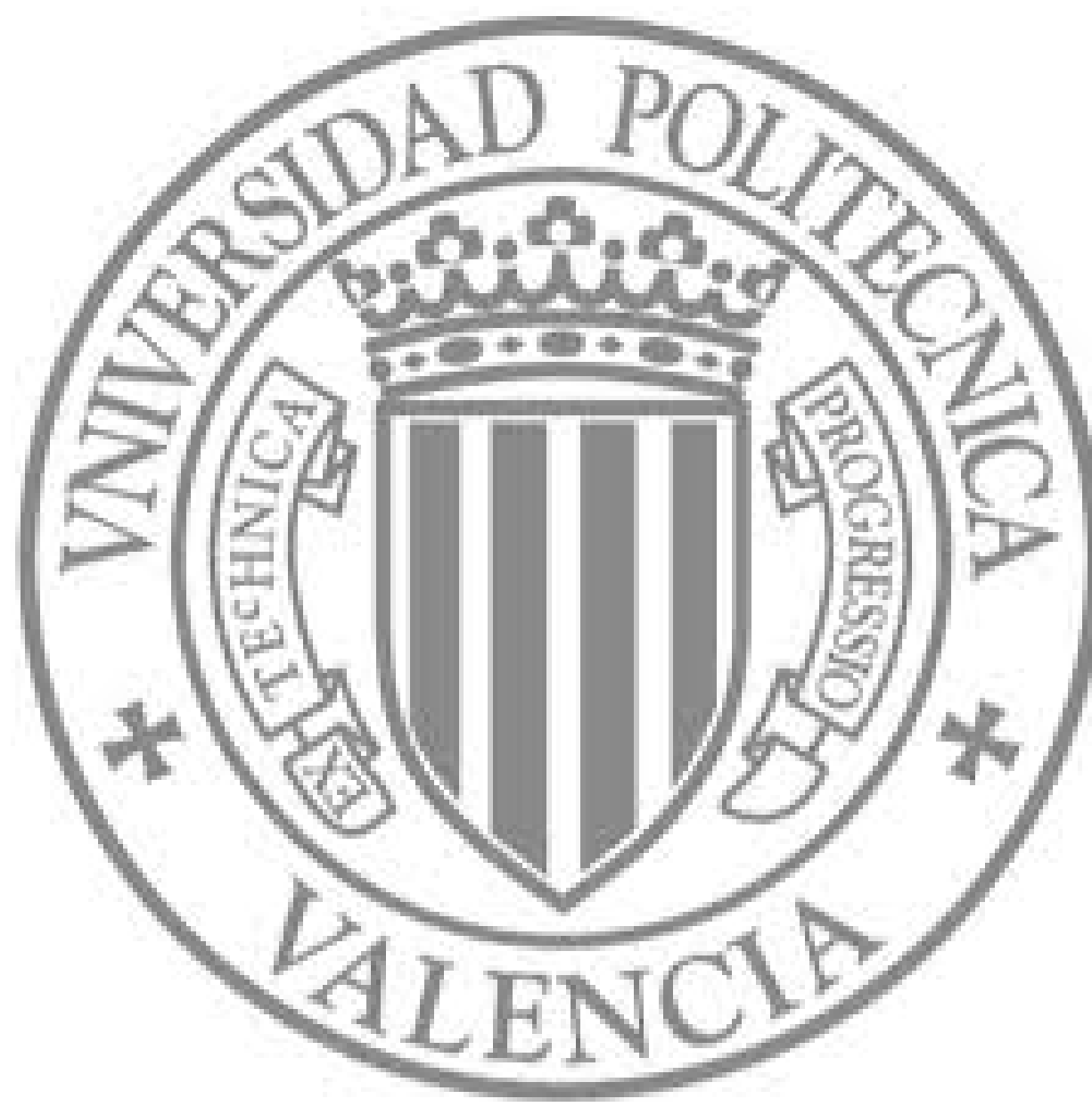

The final publication is available at

http://www.sciencedirect.com/science/article/pii/S0025540813001220

Copyright Elsevier 


\section{Crystal structure of $\mathrm{HgGa}_{2} \mathrm{Se}_{4}$ under compression}

Oscar Gomis, a, ${ }^{*}$ Rosario Vilaplana, ${ }^{a}$ Francisco Javier Manjón, ${ }^{b}$ David SantamaríaPérez, ${ }^{\mathrm{c}, \mathrm{d}}$ Daniel Errandonea, ${ }^{\mathrm{d}}$ Eduardo Pérez-González, Javier López-Solano, ${ }^{\mathrm{e}}$ Plácida Rodríguez-Hernández, ${ }^{\mathrm{e}}$ Alfonso Muñoz, ${ }^{\mathrm{e}}$ Ion Mihail Tiginyanu, ${ }^{\mathrm{f}}$ and Veaceslav Vladimir Ursaki ${ }^{\mathrm{f}}$

${ }^{a}$ Centro de Tecnologías Físicas: Acústica, Materiales y Astrofísica, MALTA Consolider Team, Universitat Politècnica de València, 46022 València, Spain

${ }^{\mathrm{b}}$ Instituto de Diseño para la Fabricación y Producción Automatizada, MALTA Consolider Team, Universitat Politècnica de València, 46022 València, Spain

${ }^{\mathrm{c}}$ Departamento de Química Física I, Universidad Complutense de Madrid, MALTA Consolider Team, Avenida Complutense s/n, 28040 Madrid, Spain

${ }^{\mathrm{d}}$ Departamento de Física Aplicada-ICMUV, MALTA Consolider Team, Universidad de Valencia, Edificio de Investigación, C/Dr. Moliner 50, Burjassot, 46100 Valencia, Spain

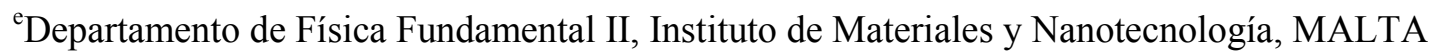
Consolider Team, Universidad de La Laguna, 38205 Tenerife, Spain

${ }^{\mathrm{f}}$ Institute of Applied Physics, Academy of Sciences of Moldova, 2028 Chisinau, Moldova

* Corresponding author. Tel.: +34 96652 8426; fax: +34 966528485.

E-mail address: osgohi@fis.upv.es (Oscar Gomis)

Dr. Oscar Gomis

Departamento de Física Aplicada

Escuela Politécnica Superior de Alcoy

Universitat Politècnica de València

Placeta Ferrandiz Carbonell 2

03802 Alcoy (Alicante)

Spain 
31

\section{Keywords:}

45 A. Chalcogénides

46

47

48

49

50

51

52

53

54

55

46 C. High-pressure

47 C. X-ray diffraction

8 D. Crystal structure

9 D. Phase transitions

We report on high-pressure $\mathrm{x}$-ray diffraction measurements up to $17.2 \mathrm{GPa}$ in mercury digallium selenide $\left(\mathrm{HgGa}_{2} \mathrm{Se}_{4}\right)$. The equation of state and the axial compressibilities for the low-pressure tetragonal phase have been determined and compared to related compounds. $\mathrm{HgGa}_{2} \mathrm{Se}_{4}$ exhibits a phase transition on upstroke towards a disordered rock-salt structure beyond $17 \mathrm{GPa}$, while on downstroke it undergoes a phase transition below 2.1 GPa to a phase that could be assigned to a metastable zinc-blende structure with a total cation-vacancy disorder. Thermal annealing at low- and high-pressure shows that kinetics plays an important role on pressure-driven transitions.

3 PACS numbers: $61.05 . \mathrm{cp}, 61.50 . \mathrm{Ks}, 62.50 .-\mathrm{p}, 64.70 . \mathrm{kg}$ 


\section{Introduction}

Mercury digallium selenide $\left(\mathrm{HgGa}_{2} \mathrm{Se}_{4}\right)$ is one of the less studied adamantinetype $A^{\mathrm{II}} B_{2}{ }^{\mathrm{III}} X_{4}{ }^{\mathrm{VI}}$ ordered-vacancy compounds (OVCs). It crystallizes in the tetragonal defect-chalcopyrite (DC) structure with space group (SG) I-4, Z=2 [see Fig. 1(a)]. Adamantine OVCs are tetrahedrally-coordinated semiconductors which have an unoccupied cationic site $[\mathbf{1}, \mathbf{2}]$. The presence of vacancies results in a complex physics and explains why OVCs have been scarcely studied. A common feature of them is that they have several non-equivalent tetrahedrally-coordinated cations resulting in a distortion of the crystal lattice from the cubic symmetry. This fact, their anisotropy, and their band-gap energies make them suitable for many technological applications $[3,4]$. High-pressure (HP) studies on $A^{\mathrm{II}} B_{2}{ }^{\mathrm{III}} X_{4}{ }^{\mathrm{VI}}$ compounds are receiving increasing attention in the last years [3-20]. In particular, ternary selenide compounds have been recently studied $[3,4,6-10,12-20]$. However, to our knowledge, only one work has been devoted to $\mathrm{HgGa}_{2} \mathrm{Se}_{4}$ [3], being focused on optical properties. In order to improve the knowledge of the HP behaviour of $A \mathrm{Ga}_{2} \mathrm{Se}_{4}$ compounds, we report here synchrotron XRD measurements in $\mathrm{HgGa}_{2} \mathrm{Se}_{4}$. In particular, we show evidence of the presence of two new phases. They can be probably assigned to a disordered rock-salt (DR) structure (SG: Fm-3m, Z=1) [see Fig. 1(b)] and a disorder zinc-blende (DZ) structure (SG: F43m, $Z=1)$ [see Fig. 1(c)].

\section{Experimental section}

Single crystals of $\mathrm{DC}-\mathrm{HgGa}_{2} \mathrm{Se}_{4}$ have been grown from its constituents $\mathrm{HgSe}$ and $\mathrm{Ga}_{2} \mathrm{Se}_{3}$ by chemical vapor transport method using iodine as a transport agent [21]. Chemical and structural analyses have shown the stoichiometric composition of the 
crystals and no spurious phases have been observed. Ambient pressure x-ray diffraction and Raman spectroscopy confirmed that our sample has a DC-type structure.

We have carried out a HP angle-dispersive powder x-ray diffraction (XRD) experiment at room temperature. This experiment was performed up to $17.2 \mathrm{GPa}$ at beamline ID27 of ESRF using a monochromatic beam $(\lambda=0.3738 \AA)$ with a beam diameter of $5 \mu \mathrm{m}$ full-width at half maximum. In this experiment samples were loaded in a modified Merrill-Basset diamond anvil cell (DAC) allowing access to an angular range of $2 \theta=25^{\circ} . \mathrm{HgGa}_{2} \mathrm{Se}_{4}$ powder was placed in the $150 \mu \mathrm{m}$-diameter hole of a stainless-steel gasket pre-indented to a thickness of $50 \mu \mathrm{m}$. XRD images were collected using a MARCCD detector located at $238 \mathrm{~mm}$ away from the sample and then integrated and corrected for distortions using FIT2D [22]. The typical acquisition time was $10 \mathrm{~s}$. In this case samples were loaded in the DAC with $\mathrm{MgO}$ which was used both as the pressure-transmitting medium (PTM) and as pressure marker using its equation of state (EOS): $\mathrm{B}_{0}=154.7 \mathrm{GPa}$, and $\mathrm{B}_{0}{ }^{\prime}=4.69$ [23]. We select the use of this pressure medium because the non-hydrostatic conditions thus generated favor the occurrence of phase transitions $[\mathbf{2 4}, \mathbf{2 5}]$. The indexing and refinement of the powder patterns were performed using the UNITCELL [26], POWDERCELL [27] and GSAS program packages [28, 29].

\section{Results and discussion} (1)

Figure 2 shows selected XRD patterns of $\mathrm{DC}-\mathrm{HgGa}_{2} \mathrm{Se}_{4}$ from ambient pressure till 17.2 $\mathrm{GPa}$ obtained in our experiment on increasing and decreasing pressure. Asterisks mark the peaks corresponding to $\mathrm{MgO}$. Table 1 summarizes the lattice parameters and atomic positions of $\mathrm{DC}-\mathrm{HgGa}_{2} \mathrm{Se}_{4}$, obtained at 1 bar from a Rietveld 
refinement of our XRD pattern. The refined parameters were: the scale factor, lattice parameters, profile coefficients, fractional coordinates of the Se anion, and the overall displacement factor. The background was subtracted previously. Our results agree with those of Refs. 30 and $\mathbf{3 1}$ which are also shown in Table $\mathbf{1}$ for comparison.

In Fig. 2 diffractograms from 1 bar to $17.2 \mathrm{GPa}$ on upstroke correspond to the low-pressure tetragonal DC phase and show that diffraction peaks move to higher angles as pressure increases, thus indicating that the interplanar distances decrease. It is also observed that peak widths of the $\mathrm{DC}-\mathrm{HgGa}_{2} \mathrm{Se}_{4}$ phase increase gradually above 7 GPa which implies that hydrostatic conditions are deteriorating with increasing pressure. On the other hand, in general, $\mathrm{HgGa}_{2} \mathrm{Se}_{4}$ peaks move faster than those of $\mathrm{MgO}$ as can be seen from the (111) $\mathrm{MgO}$ Bragg reflection located at $8.93^{\circ}$ at $7.1 \mathrm{GPa}$. This reflection is overtaken at $10.1 \mathrm{GPa}$ by the (211) $\mathrm{DC}-\mathrm{HgGa}_{2} \mathrm{Se}_{4}$ Bragg reflection located at $8.71^{\circ}$ at 1 bar. This fact is a consequence of the different compressibility of $\mathrm{MgO}$ and $\mathrm{HgGa}_{2} \mathrm{Se}_{4}$. At 17.2 $\mathrm{GPa}$ all peaks broaden considerably and two new broad peaks appear (see + marks). We have interpreted this result as a signature of the onset of a non-reversible phase transition. In order to release strain in the sample at $17.2 \mathrm{GPa}$ we annealed the sample (393 K during 1 hour) using an external heater [32]. After thermal treatment, pressure decreased slightly $(16.2 \mathrm{GPa})$ and the new XRD pattern showed some remnant peaks from the initial DC phase and a major proportion of a new phase that can be assigned to the DR structure phase previously proposed for related compounds [10, 16-19]. According to the relation of intensities of Bragg peaks of both phases [33], we can estimate that an $85 \%$ of the sample was transformed to the HP phase. Unfortunately, the DAC we used prevented us from going to higher pressures. A Rietveld refinement of the difractogram measured at $16.2 \mathrm{GPa}$ at the upstroke which shows the coexistence of the DR and DC phases along with $\mathrm{MgO}$ is included in Fig. 2. 
131 The multiphase Rietveld gives the same result for the amount of sample transformed to the HP phase. Table 2 summarizes the crystallographic parameters of DR- $\mathrm{HgGa}_{2} \mathrm{Se}_{4}$ at 16.2 GPa. The quality of the structural refinement is similar for the pattern collected at 16.2 GPa after annealing than for the pattern collected at 1 bar (see Fig. 2 and residuals in Tables 1 and 2).

On decompression we took several XRD patterns showing the coexistence of DR and DC phases till 5.7 GPa, with the DR phase being in all cases the dominant one. Below 5.7 GPa the peaks of the HP phase disappear and broad XRD peaks appear as shown in the spectrum of Fig. 2 at $2.1 \mathrm{GPa}$ (solid line) on downstroke. As we will discuss below, apparently the changes observed in the XRD pattern can be assigned to a transition to a phase different than the low- and high-pressure phases previously described. The XRD patterns of this new phase can be attributed to a DZ structure. Again, in order to release strain, we annealed the recovered sample at $2.1 \mathrm{GPa}$ (393 K during 30 minutes). After thermal treatment, pressure decreased to $1.5 \mathrm{GPa}$ and the new XRD pattern showed a well-defined tetragonal structure, which proved to be similar to the structure of the low-pressure phase, but with broader peaks that could evidence some degree of disorder in the sample. A plausible hypothesis that can explain this phenomenon is that the cation and vacancy disorder caused during the DC-to-DR transition cannot be reordered upon decompression resulting in the appearance of a metastable DZ phase on decompression. The additional energy provided by the thermal annealing helps to reduce disorder and relax stresses favoring the recovery of the

152 thermodynamically stable low-pressure phase. A DZ structure has been already found in from the DR structure. 
As mentioned above, a detailed study of the XRD pattern obtained on

downstroke at 2.1 GPa before annealing evidence the presence of a possible metastable

157 phase with DZ structure. In Figure 2 it is shown the comparison of the diffraction pattern measured upon decompression at $2.1 \mathrm{GPa}$ (solid line) and the calculated pattern (dash-dotted line) for a DZ phase. A lattice parameter of 5.45(1) $\AA$ provides the best matching to the new peaks assigned to the DZ structure. To facilitate comparison of both diffractograms, the background was subtracted in the measured one and the simulated diffractogram was modeled with profile parameters obtained by comparison to the measured one using the Powdercell software. The resemblance of both experimental and calculated diffractograms is quite good. The broad aspect of the allowed zinc-blende diffraction Bragg reflections is likely to be a consequence of the complete disorder of cations and vacancies at the only cation site (4a Wyckoff position) in the metastable zinc-blende structure (see Table 2).

We would like to mention here that because of the broad bands of the diffraction pattern measured at 2.1 GPa and the fact that some of the Bragg peaks attributed to the DZ phase have quite a low intensity a structural refinement cannot be performed. Note also, that because the high symmetry of both the DR and DZ structures a maximum of 6 to 8 Bragg peaks of them can be detected working in a diamond-anvil cell even using short wavelength like in this work. This fact also precludes the performance of a kosher structural refinement even for simple structures like DR and DZ in which neither atom possesses any internal degree of freedom. In previous works, both phases have been 176 identified in related compounds with less than six reflections, but not proper structural 177 refinements were carried out $[10, \mathbf{1 7}, \mathbf{1 9}]$ All these facts indicate that in the future it will be necessary to carry out single-crystal XRD experiments [34] in order to properly 
interesting issue to explore in the future is the role of kinetics. The fact that thermal annealing is requested for completion of the transitions triggered by compression or decompression shows that kinetical barriers could be relevant in the studied transitions.

On the other hand, our observation of a tetragonal phase at $1.5 \mathrm{GPa}$ after heating a decompressed sample with DZ structure at $2.1 \mathrm{GPa}$ is compatible with previous studies that show a recrystallization of the DC structure in $\mathrm{Zn}_{1-\mathrm{x}} \mathrm{Mn}_{\mathrm{x}} \mathrm{Ga}_{2} \mathrm{Se}_{4}$ after a moderate heating of samples with the defect stannite structure, which already has some degree of disorder, above $300^{\circ} \mathrm{C}$ in vacuum and decreasing temperature in a controlled way [35, 36].

Figure 3 shows the pressure dependence of the lattice parameters for DC$\mathrm{HgGa}_{2} \mathrm{Se}_{4}$ from our experiment. The axial compressibilities for $a$ and $c$ axes at zero 191 pressure, defined as $\kappa_{x}=\frac{-1}{x} \frac{\partial x}{\partial P}$ and obtained by fitting of a Murnaghan EOS to experimental data [37], are $\kappa_{a}=9(2) \cdot 10^{-3} \mathrm{GPa}^{-1}$ and $\kappa_{c}=5(1) \cdot 10^{-3} \mathrm{GPa}^{-1}$. It can be observed that there is an anisotropy in the axial compression being the $a$ axis more compounds $[10,16,18,19,38]$.

Figure 4 shows the volume of the DC phase vs. pressure plot obtained from our experiment (circles). Experimental data for the DR phase on downstroke and the DZ phase at 2.1 GPa are shown as diamonds and squares, respectively. We have fitted our volume vs. pressure data for the DC phase with a third order Birch-Murnaghan EOS [39]. The fitting of the data of Fig. 4 (dashed line) with a volume at zero pressure fixed at a value of $\mathrm{V}_{0}=352.70(16) \AA^{3}$ (the measured value at ambient pressure) and the bulk modulus pressure derivative at zero pressure fixed at a value of $\mathrm{B}_{0}{ }^{\prime}=4$ gives a bulk modulus of $\mathrm{B}_{0}=52(2) \mathrm{GPa}$. The EOS parameters are summarized in Table 3 together with parameters obtained from a different experiment carried out with a laboratory 
diffractometer using methanol-ethanol as pressure transmitting medium to reduce deviatoric stresses and in a reduced pressure range (13.2 GPa) to avoid the influence of precursor effects [40] of the pressure-driven transition on the structure of the lowpressure phase [38]. If we compare the obtained value for $\mathrm{B}_{0}$ when $\mathrm{B}_{0}$ ' is fixed to 4 for both experiments, it can be seen that the $\mathrm{B}_{0}$ for the experiment when $\mathrm{MgO}$ is used as PTM is about $16 \%$ greater than that $\mathrm{B}_{0}$ obtained when methanol-ethanol is used as PTM. This result confirms the overestimation of $\mathrm{B}_{0}$ under non-hydrostatic conditions noted in previous works $[\mathbf{2 4}, \mathbf{2 5}, \mathbf{4 1}, \mathbf{4 2}, \mathbf{4 3}]$. Finally, we note that the obtained value for $\mathrm{B}_{0}$ in $\mathrm{DC}-\mathrm{HgGa}_{2} \mathrm{Se}_{4}$ from our experiment with $\mathrm{MgO}$ as PTM is similar to that obtained for DC-CdGa $\mathrm{Se}_{4}\left(\mathrm{~B}_{0}=41.5(2) \mathrm{GPa}\right)$ [10], DC- $\mathrm{MnGa}_{2} \mathrm{Se}_{4}\left(\mathrm{~B}_{0}=44(2) \mathrm{GPa}\right)$ [16], DC$\mathrm{CdAl}_{2} \mathrm{Se}_{4}\left(\mathrm{~B}_{0}=52.1 \mathrm{GPa}\right)$ [17], and DS- $\mathrm{ZnGa}_{2} \mathrm{Se}_{4}\left(\mathrm{~B}_{0}=47(2) \mathrm{GPa}\right)$ [18].

As regards the DR phase, it can be observed from Fig. 4 that it is less compressible than the $\mathrm{DC}$ phase. We have estimated a relative volume change per formula unit of $-2.2 \%$ at $16.2 \mathrm{GPa}$, thus indicating that the DC to DR phase transition is a first-order phase transition of reconstructive nature. A fit of our experimental volume vs. pressure data for the DR phase with a Birch-Murnaghan EOS with $\mathrm{B}_{0}$ ' fixed to 4 gives a bulk modulus of $\mathrm{B}_{0}=103(6) \mathrm{GPa}$ and a volume at zero pressure $\mathrm{V}_{0}=159.9(8)$ $\AA^{3}$. The greater value for the $\mathrm{B}_{0}$ of the DR phase in comparison to that of the DC phase confirms the lower compressibility of the HP phase. The same result is found for other OVCs like $\mathrm{MnGa}_{2} \mathrm{Se}_{4}, \mathrm{CdAl}_{2} \mathrm{~S}_{4}$ and $\mathrm{ZnGa}_{2} \mathrm{Se}_{4}$ [16, 18, 19]. If we compare the normalized volumes of the DC, DR, and DZ phases at $2.1 \mathrm{GPa}$ it is found that the volume of the DZ phase $\left[324(2) \AA^{3}\right]$ is between those of the DC [338(3) $\left.\AA^{3}\right]$ and DR [314(2) $\left.\AA^{3}\right]$ phases. In the comparison, the volume for the DR phase at $2.1 \mathrm{GPa}$ has been extrapolated by using the EOS, and in the cases of the DR and DZ volume was normalized multiplying by two. The volume of the three phases decreases in the 
sequence DC $>$ DZ $>$ DR which suggest that the compressibility of the DZ structure should be in between those of the other two phases since the packing efficiency of DZ is in between those of DC and DR.

Now we will analyze the evolution of the $c / a$ ratio with pressure in DC$\mathrm{HgGa}_{2} \mathrm{Se}_{4}$ since the tetragonal distortion, $\delta=2-c / a$, could give important information about the behavior of the sample on compression. Inset of Fig. 4 shows the pressure dependence of the $c / a$ ratio vs. pressure. It can be observed that $c / a$ increases with increasing pressure from 1.89 at ambient pressure to 1.94 at $17.3 \mathrm{GPa}$. A similar experimental pressure dependence of the $c / a$ ratio has been found in $\mathrm{CdGa}_{2} \mathrm{Se}_{4}[\mathbf{1 0 ]}$, $\mathrm{MnGa}_{2} \mathrm{Se}_{4}$ [16], $\mathrm{CdGa}_{2} \mathrm{~S}_{4}$ [18], $\mathrm{HgAl}_{2} \mathrm{Se}_{4}$ [19], and in $\mathrm{HgGa}_{2} \mathrm{Se}_{4}$ [38] under better hydrostatic conditions than here. It is noteworthy that $A \mathrm{Ga}_{2} X_{4}$ compounds ( $A=\mathrm{Mn}, \mathrm{Zn}$, $\mathrm{Cd}, \mathrm{Hg} ; X=\mathrm{S}, \mathrm{Se}$ ) with tetragonal $\mathrm{DC}$ structure at ambient pressure have $c / a$ values close to $1.90[\mathbf{1 0}, \mathbf{1 6}, \mathbf{3 8}, \mathbf{4 4}]$, while those with tetragonal defect stannite structure like $\mathrm{ZnGa}_{2} \mathrm{Se}_{4}$ or $\mathrm{ZnGa}_{2} \mathrm{~S}_{4}$, which have already some cation disorder, have $c / a$ ratios close to 1.98 at ambient pressure $[\mathbf{1 8}, \mathbf{4 5}$. Furthermore, a c/a ratio very close to 2, or equivalently a very small tetragonal distortion of the tetragonal phase, has been considered up to now as a measure of complete cation-vacancy disorder [31, 44]. Therefore, our results show that $\mathrm{DC}-\mathrm{HgGa}_{2} \mathrm{Se}_{4}$, like other DC compounds $[\mathbf{4}, \mathbf{1 0}, \mathbf{1 6}]$, tends to a more symmetrical structure on compression prior to undergoing the phase transition to the DR structure at $17.2 \mathrm{GPa}$.

To conclude we would like to comment on the different coordination found on the DC, DR, and DZ structures shown in Fig 1. The low pressure DC phase has fourfold coordination where cations are tetrahedrally-coordinated while anions are surrounded by three cations and a vacancy. The high pressure DR phase has six-fold coordination where cations and anions are octahedrally-coordinated. In this way, the 
255 phase transition implies an increase of the symmetry of the crystal and is accompanied 256 by a change of coordination of the cations from tetrahedral to octahedral. On the other 257 hand, the metastable DZ phase has again four-fold coordination as the original DC 258 phase.

\section{Summary}

We have performed XRD measurements in defect chalcopyrite $\mathrm{HgGa}_{2} \mathrm{Se}_{4}$ under compression. The experiments show that the pressure dependence of the volume and lattice parameters of $\mathrm{DC}-\mathrm{HgGa}_{2} \mathrm{Se}_{4}$ behaves in a similar way to other adamantine OVCs. The axial compressibilities and the equation of state of $\mathrm{HgGa}_{2} \mathrm{Se}_{4}$ have been obtained for the tetragonal DC structure under non-hydrostatic conditions. It is observed that the tetragonal structures of OVCs tend to become more symmetric under compression irrespective of the conditions of hydrostaticity. A non-reversible phase transition to the disordered rock-salt phase on increasing pressure has been found. On decreasing pressure the sample was found to undergo a phase transition to a metastable structure that might be attributed to a disordered zinc-blende structure. Apparently kinetics plays an important role on the occurrence of the reported phase transitions. This is evidenced by the fact that thermal annealing favors the occurrence of phase transitions.

\section{Acknowledgements}

275 This study was supported by the Spanish government MEC under Grants No:

276 MAT2010-21270-C04-01/03/04 and CTQ2009-14596-C02-01, by the Comunidad de 277 Madrid and European Social Fund (S2009/PPQ-1551 4161893), by MALTA Consolider Ingenio 2010 project (CSD2007-00045), and by the Vicerrectorado de Investigación y 
279 Desarrollo of the Universidad Politécnica de Valencia (UPV2011-0914 PAID-05-11 280 and UPV2011-0966 PAID-06-11). E.P-G., J.L-S., A.M., and P.R-H. acknowledge 281 computing time provided by Red Española de Supercomputación (RES) and MALTA282 Cluster.

283 
285 [1] A. MacKinnon, in Tables of Numerical Data and Functional Relationships in 286 Science and Technology, edited by O. Madelung, M. Schulz, and H. Weiss, Landolt287 Börnstein New Series, Group III, Vol. 17, pt. h (Springer-Verlag, Berlin, 1985) p. 124. 288 [2] J.E. Bernard and A. Zunger, Phys. Rev. B 37 (1988) 6835.

289 [3] F.J. Manjón, O. Gomis, P. Rodríguez-Hernández, E. Pérez-González, A. Muñoz, D. 290 Errandonea, J. Ruiz-Fuertes, A. Segura, M. Fuentes-Cabrera, I. Tiginyanu, and V.V. 291 Ursaki, Phys. Rev. B 81 (2010) 195201.

292 [4] O. Gomis, R. Vilaplana, F. J. Manjón, E. Pérez-González, J. López-Solano, P. 293 Rodríguez-Hernández, A. Muñoz, D. Errandonea, J. Ruiz-Fuertes, A. Segura, D. 294 Santamaría-Pérez, I. M. Tiginyanu, and V.V. Ursaki, Journal of Appl. Phys. 111 (2012) 295013518.

296

[5] I. I. Burlakov, Y. Raptis, V. V. Ursaki, E. Anastassakis, and I. M. Tiginyanu, Solid 297 State Commun. 101 (1997) 377.

298

[6] J. González, R. Rico, E. Calderón, M. Quintero, and M. Morocoima, phys. stat. sol. 299 (b) 211 (1999) 45 .

300

[7] V.V. Ursaki, I.I. Burlakov, I.M. Tiginyanu, Y.S. Raptis, E. Anastassakis, and A. 301 Anedda, Phys. Rev. B 59 (1999) 257.

302

[8] M. Fuentes-Cabrera and O.F. Sankey, J. Phys.: Condens. Matter 13, (2001) 1669.

303

[9] M. Fuentes-Cabrera, J. Phys.: Condens. Matter 13 (2001) 10117.

304

[10] A. Grzechnik, V.V. Ursaki, K. Syassen, I. Loa, I.M. Tiginyanu, and M. Handfland, 305 J. Solid State Chem. 160, (2001) 205. 
306 [11] T. Mitani, S. Onari, K. Allakhverdiev, F. Gashimzade, and T. Kerimova, phys. stat. sol. (b) 223 (2001) 287.

[12] A. Tatsi, D. Lampakis, E. Liarokapis, S.A. López, L. Martínez, and W. Giriat, High 309 Press. Res. 22 (2002) 89.

[13] I. M. Tiginyanu, V.V. Ursaki, F.J. Manjón, and V.E. Tezlevan, J. Phys. Chem. 311 Solids 64, (2003) 1603.

[14] T. Mitani, T. Naitou, K. Matsuishi, S. Onari, K. Allakhverdiev, F. Gashimzade, and

T. Kerimova, phys. stat. sol. (b) 235 (2003) 321.

[15] K. Allakhverdiev, F. Gashimzade, T. Kerimova, T. Mitani, T. Naitou, K. 315 Matsuishi, and S. Onari, J. Phys. Chem. Solids 64, (2003) 1597.

[16] J. Marquina, Ch. Power, P. Grima, M. Morocoima, M. Quintero, B. couzinet, J.C.

Chervin, P. Munsch, and J. González, J. Appl. Phys. 100 (2006) 093513.

[17] S. Meenakshi, V. Vijyakumar, B.K. Godwal, A. Eifler, I. Orgzall, S. Tkachev, and

H.D. Hochheimer, J. Phys. Chem. Solids 67 (2006) 1660.

320

[18] D. Errandonea, R.S. Kumar, F.J. Manjón, V.V. Ursaki, and I.M. Tiginyanu, J.

321 Appl. Phys. 104 (2008) 063524.

322

[19] S. Meenakshi, V. Vijyakumar, A. Eifler, and H.D. Hochheimer, J. Phys. Chem. 323 Solids 71 (2010) 832.

324

[20] P. Singh, M. Sharma, U.P. Verma, and P. Jensen, Z. Kristallogr. 225 (2010) 508.

325

[21] I.M. Tiginyanu, N. A. Modovyan, and O. D. Stoika, Fiz. Tverd. Tela 34 (1992) 967 (1992); idem, Sov. Phys. Solid State 43 (1992) 527. 
327 [22] A. P. Hammersley, S. O. Svensson, M. Hanfland, A. N. Fitch, and D. Häusermann, 328 High Pressure Research, 14 (1996) 235.

329 [23] P. I. Dorogokupets, and A. Dewaele, High Pressure Research 27 (2007) 431.

330 [24] J. Ruiz-Fuertes, D. Errandonea, R. Lacomba-Perales, A. Segura, J. González, F. 331 Rodríguez, F. J. Manjón, S. Ray, P. Rodríguez-Hernández, A. Muñoz, Zh. Zhu, and C. 332 Y. Tu, Phys. Rev. B, 81 (2010) 224115.

333 [25] O. Gomis, J. A. Sans, R. Lacomba-Perales, D. Errandonea, Y. Meng, J. C. Chervin, 334 and A. Polian, Phys. Rev. B, 86 (2012) 054121.

335 [26] T. J. B. Holland, and S. A. T. Redfern, Mineralogical Magazine, 61 (1997) 65.

336 [27] W. Kraus and G. Nolze, J. Appl. Crystallogr. 29 (1996) 301.

337 [28] A. C. Larson and R. B. von Dreele, LANL Report 86-748, 2004 (unpublished).

338 [29] B. H. Toby, J. Appl. Cryst. 34 (2001) 210.

339 [30] H. Hahn, G. Frank, W. Klingler, A.D. Stoerger, and G. Stoerger, Zeitschrift fuer 340 Anorganische und Allgemeine Chemie 279 (1955) 241.

341 [31] L. Gastaldi, M.G. Simeone, and S. Viticoli, Solid State Commun. 55 (1985) 605.

342 [32] D. Errandonea, D. Martínez-García, A. Segura, A. Chevy, G. Tobias, E. Canadell, 343 and P. Ordejon, Phys. Rev. B 73 (2006) 235202.

344 [33] D. Errandonea, R. Boehler, S. Japel, M. Mezouar, and L. R. Benedetti, Phys. Rev. 345 B 73 (2006) 092106. 
346

347

348

349

350

351

352

353

354

355

356

357

358

359

360

361

362

363

364

365

366

367

[34] J. Ruiz-Fuertes, A. Friedrich, J. Pellicer-Porres, D. Errandonea, A. Segura, W. Morgenroth, E. Haussühl, C.-Y. Tu, and A. Polian, Chemestry of Materials 23 (2011) 4220.

[35] P. Alonso-Gutiérrez, M.L. Sanjuán, and M.C. Morón, phys. Stat. sol. (c) 6 (2009) 1182. P. Alonso-Gutiérrez, Ph. D. thesis, University of Zaragoza (2009), unpublished.

[36] D. Caldera, M. Morocoima, M. Quintero, C. Rincón, R. Casanova, and P. Grima, Solid State Commun. 151 (2011) 212.

[37] M. D. Frogley, J. L. Sly, and D. J. Dunstan, Phys. Rev. B 58 (1998) 12579.

[38] O. Gomis, R. Vilaplana, F.J. Manjón, D. Santamaría-Pérez, D. Errandonea, E. Pérez-González, J. López-Solano, P. Rodríguez-Hernández, A. Muñoz, I. M. Tiginyanu, and V. V. Ursaki, submitted to Journal of Applied Physics.

[39] F. Birch, J. Geophys. Res. 83 (1978) 1257.

[40] E. Bandiello, D. Errandonea, D. Martinez-Garcia, D. Santamaria-Perez, and F. J. Manjon, PRB 85 (2012) 024108.

[41] H.Z Liu, Y. Ding, M. Somayazulu, J. Qian, J. Shu, D. Häusermann, and H.K. Mao, Phys. Rev. B 71 (2005) 212103.

[42] H.Z. Liu, J.Z. Hu, J.F. Shu, D. Häusermann, and H.K. Mao, Appl. Phys. Lett. 85 (2004) 1973.

[43] D. Santamaría-Pérez, L. Gracia, G. Garbarino, A. Beltrán, R. Chulia-Jordan, O. Gomis, D. Errandonea, Ch. Ferrer-Roca, D. Martínez-García, and A. Segura, Phys. Rev. B 84 (2011) 054102.

[44] L. Garbato, F. Ledda, and A. Rucci, Prog. Cryst. Growth Charact. 15 (1987) 1. 
[45] C. K. Lowe-Ma and T.A. Vanderah, Acta Cryst. C 47 (1991) 919.

369 
Table 1. Experimental crystallographic parameters of tetragonal (I-4, Z=2) $\mathrm{HgGa}_{2} \mathrm{Se}_{4}$ at

371 room conditions. The residuals for the Rietveld refinement are $R_{p}=11 \%$ and $R_{w p}$ $372=16.4 \%$.

373

\begin{tabular}{|c|c|c|c|}
\hline & $\begin{array}{c}\text { X-ray } \\
\text { diffraction }^{a}\end{array}$ & $\begin{array}{c}\text { X-ray } \\
\text { diffraction }^{b}\end{array}$ & $\begin{array}{c}\text { X-ray } \\
\text { diffraction }^{c}\end{array}$ \\
\hline$a(\AA)$ & $5.711(1)$ & 5.715 & $5.693(1)$ \\
\hline$c(\AA)$ & $10.814(1)$ & 10.78 & $10.826(4)$ \\
\hline $\begin{array}{c}\mathrm{Hg} \\
\text { site: } 2 a\end{array}$ & $\begin{array}{l}x=0 \\
y=0 \\
z=0\end{array}$ & $\begin{array}{l}x=0 \\
y=0 \\
z=0\end{array}$ & $\begin{array}{l}x=0 \\
y=0 \\
z=0\end{array}$ \\
\hline $\begin{array}{l}\mathrm{Ga}(1) \\
\text { site: } 2 b\end{array}$ & $\begin{array}{l}x=0 \\
y=0 \\
z=0.5\end{array}$ & $\begin{array}{l}x=0 \\
y=0 \\
z=0.5\end{array}$ & $\begin{array}{l}x=0 \\
y=0 \\
z=0.5\end{array}$ \\
\hline $\begin{array}{l}\mathrm{Ga}(2) \\
\text { site: } 2 c\end{array}$ & $\begin{array}{l}x=0 \\
y=0.5 \\
z=0.25\end{array}$ & $\begin{array}{l}x=0 \\
y=0.5 \\
z=0.25\end{array}$ & $\begin{array}{l}x=0 \\
y=0.5 \\
z=0.25\end{array}$ \\
\hline $\begin{array}{l}\text { Vacancy } \\
\text { site: } 2 d\end{array}$ & $\begin{array}{l}x=0 \\
y=0.5 \\
z=0.75\end{array}$ & $\begin{array}{l}x=0 \\
y=0.5 \\
z=0.75\end{array}$ & $\begin{array}{l}x=0 \\
y=0.5 \\
z=0.75\end{array}$ \\
\hline $\begin{array}{c}\mathrm{Se} \\
\text { site: } 8 g\end{array}$ & $\begin{array}{l}x=0.270(2) \\
y=0.245(5) \\
z=0.1315(6)\end{array}$ & $\begin{array}{l}x=0.25 \\
y=0.25 \\
z=0.125\end{array}$ & $\begin{array}{l}x=0.273(1) \\
y=0.2582(8) \\
z=0.1382(6)\end{array}$ \\
\hline
\end{tabular}


379 Table 2. Experimental crystallographic parameters of DR (Fm-3m, Z=1) $\mathrm{HgGa}_{2} \mathrm{Se}_{4}$ at $38016.2 \mathrm{GPa}$. The lattice parameter is $a=5.2048(5) \AA$. The residuals for the Rietveld 381 refinement are $R_{p}=9.8 \%$ and $R_{w p}=13.4 \%$. We also include the atomic positions used 382 to simulate $\mathrm{DZ}-\mathrm{HgGa}_{2} \mathrm{Se}_{4}(\mathrm{~F}-43 \mathrm{~m}, \mathrm{Z}=1)$ at $2.1 \mathrm{GPa}$ with $\mathrm{a}=5.45(1) \AA$.

\begin{tabular}{|c|c|c|c|c|}
\hline \multirow{2}{*}{} & \multicolumn{2}{|c|}{ DR-HgGa $\mathrm{Se}_{4}$} & \multicolumn{2}{c|}{ DZ- $\mathrm{HgGa}_{2} \mathrm{Se}_{4}$} \\
\hline & Wyckoff position & Site occupancy & Wyckoff position & Site occupancy \\
& & factor (S.O.F.) & & factor (S.O.F.) \\
\hline $\mathrm{Hg}$ & $4 \mathrm{a}(0,0,0)$ & 0.25 & $4 \mathrm{a}(0,0,0)$ & 0.25 \\
\hline $\mathrm{Ga}$ & $4 \mathrm{a}(0,0,0)$ & 0.5 & $4 \mathrm{a}(0,0,0)$ & 0.5 \\
\hline Vacancy & $4 \mathrm{a}(0,0,0)$ & 0.25 & $4 \mathrm{a}(0,0,0)$ & 0.25 \\
\hline Se & $4 \mathrm{~b}(1 / 2,1 / 2,1 / 2)$ & 1 & $4 \mathrm{c}(1 / 4,1 / 4,1 / 4)$ & 1 \\
\hline
\end{tabular}

384

385 
387 Table 3: Experimental (exp.) volume $\left(\mathrm{V}_{0}\right)$, bulk modulus $\left(\mathrm{B}_{0}\right)$, and its pressure 388 derivative $\left(\mathrm{B}_{0}{ }^{\prime}\right)$ for $\mathrm{DC}-\mathrm{HgGa}_{2} \mathrm{Se}_{4}$ at ambient pressure. Values were obtained by fitting 389 data to a third-order Birch-Murnaghan EOS with $\mathrm{B}_{0}$ ' fixed to 4 and $\mathrm{V}_{0}$ fixed to the 390 value measured at 1 bar. Data from Ref. 38 are also included for comparison.

\begin{tabular}{|l|l|l|l|l|}
\hline & $\mathrm{V}_{0}\left(\AA^{3}\right)$ & $\mathrm{B}_{0}(\mathrm{GPa})$ & $\mathrm{B}_{0} ’$ & References \\
\hline exp. & 352.70 & $52(2)$ & 4 (fixed) & This work \\
\hline exp. & $352.9(6)$ & $39(2)$ & $5.2(4)$ & $\mathbf{3 8}$ \\
& $351.4(5)$ & $44.9(7)$ & 4 (fixed) & \\
\hline
\end{tabular}

392

393 
Figure 1. (Color online) (a) Structure of the defect chalcopyrite (DC) $\mathrm{HgGa}_{2} \mathrm{Se}_{4}$, (b) defect rock salt (DR) $\mathrm{HgGa}_{2} \mathrm{Se}_{4}$, and (c) defect zinc blende (DZ) $\mathrm{HgGa}_{2} \mathrm{Se}_{4}$. Big light atoms are $\mathrm{Hg}$, medium dark atoms are $\mathrm{Ga}$, and small dark atoms are Se. To distinguish between nonequivalent atoms in the DC structure, the Wyckoff sites are given in parenthesis.

Figure 2. XRD patterns of $\mathrm{HgGa}_{2} \mathrm{Se}_{4}$ on upstroke up to $17.2 \mathrm{GPa}$ and downstroke to 1.5 GPa. The diffractogram measured at 1 bar at the upstroke is shown as solid circles. The calculated pattern at 1 bar obtained from a Rietveld refinement along with the residuals are shown as solid lines. A Rietveld refinement of the difractogram measured at 16.2 $\mathrm{GPa}$ at the upstroke showing the coexistence of the DR and DC phases along with $\mathrm{MgO}$ is included. The residuals are $\mathrm{R}_{\mathrm{p}}=9.8 \%$ and $\mathrm{R}_{\mathrm{wp}}=13.4 \%$. In the pattern collected at 2.1 GPa on downstroke, we show the comparison of the measured pattern (solid line) and the calculated diffractogram using Powdercell software for the defect zincblende (DZ) phase (dash-dotted line). Vertical marks indicate the Bragg reflections for the DC phase at 1 bar at the upstroke, for the $\mathrm{DR}$ and $\mathrm{DC}$ phases and $\mathrm{MgO}$ at $16.2 \mathrm{GPa}$ at the upstroke, and for the DZ phase at 2.1 GPa at the downstroke. Plus $(+)$ symbols refer to

412 reflections attributed to the disordered rocksalt phase and $\mathrm{MgO}$ reflections are marked with $*$ symbols.

414 Figure 3. Lattice parameters of the DC phase of $\mathrm{HgGa}_{2} \mathrm{Se}_{4}$ as a function of pressure.

415 Solid and empty circles refer to data from our XRD experiment on increasing and 416 decreasing pressure, respectively. Solid lines are a guide to the eye. 
417 Figure 4. Volume of the DC (circles) and DR (diamonds) phases as a function of 418 pressure. The volume of the DZ phase at $2.1 \mathrm{GPa}$ is included as square symbols. Full 419 symbols are used for upstroke and empty symbols for downstroke. Note that for 420 comparison of the three structures we have plotted twice the volume of the DR and DZ 421 phases since the unit cell of DC phase has $\mathrm{Z}=2$ while that of the DR and DZ phases has $422 \mathrm{Z}=1$. Dashed and dash-dotted lines are the result of the EOS fit for the DC and DR 423 phases of our experiment. Inset: Evolution of the c/a ratio of the DC phase as a function 424 of pressure for our experiments (circles). Dashed line is a linear fit to experimental data. 
Figure 1.

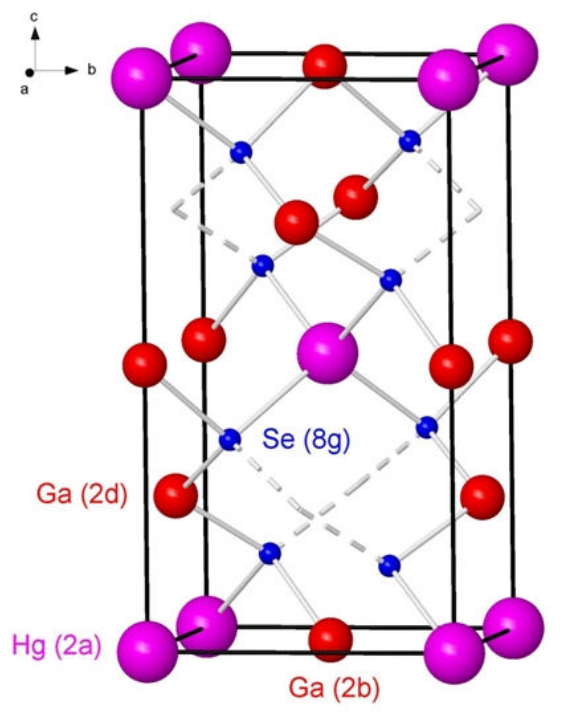

(a)

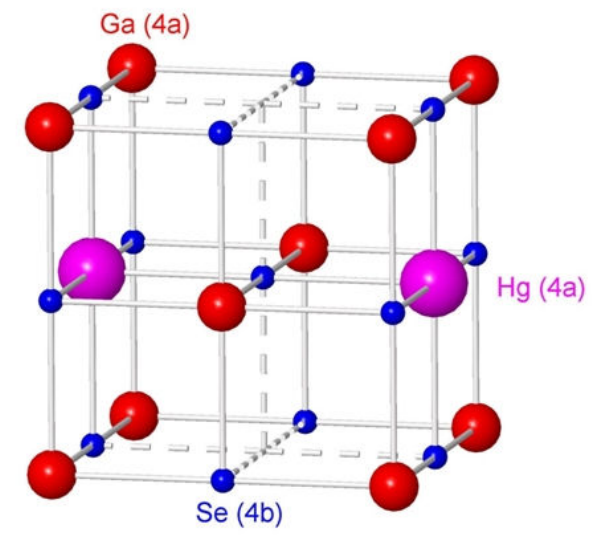

(b)

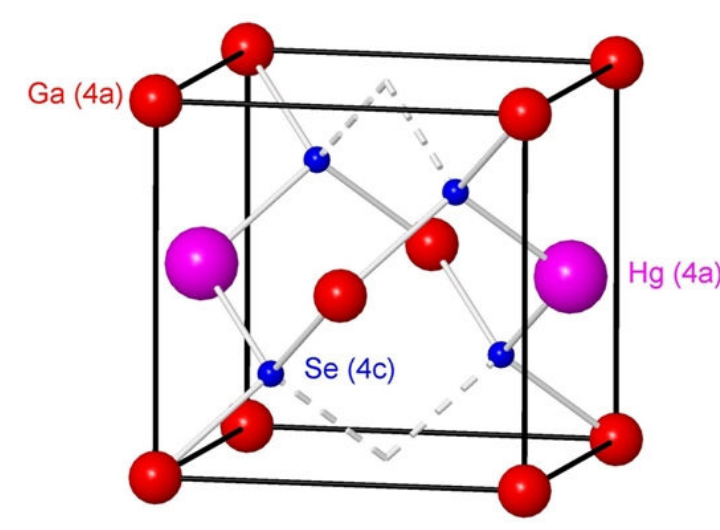

(c) 
Figure 2

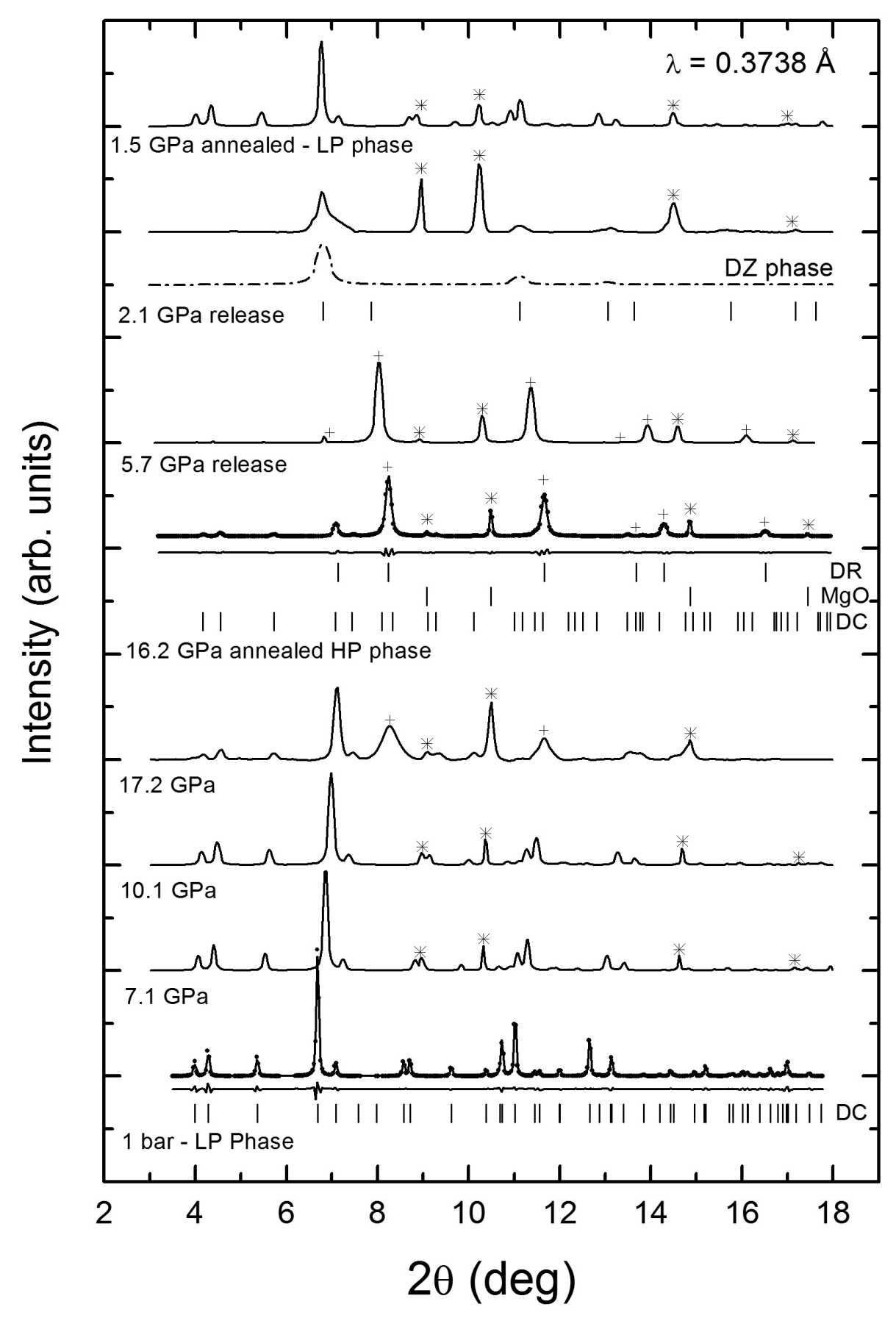


Figure 3

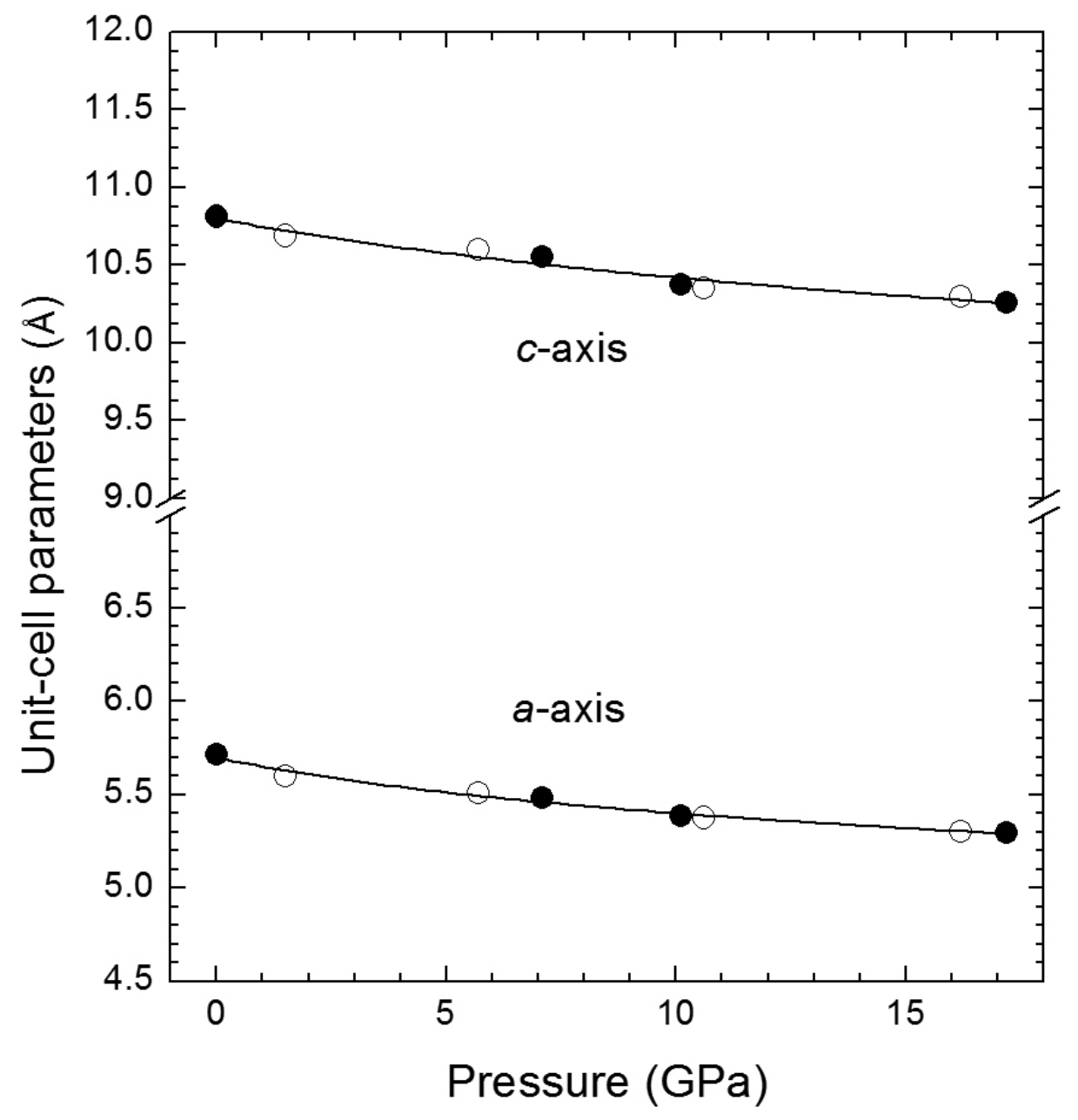


Figure 4

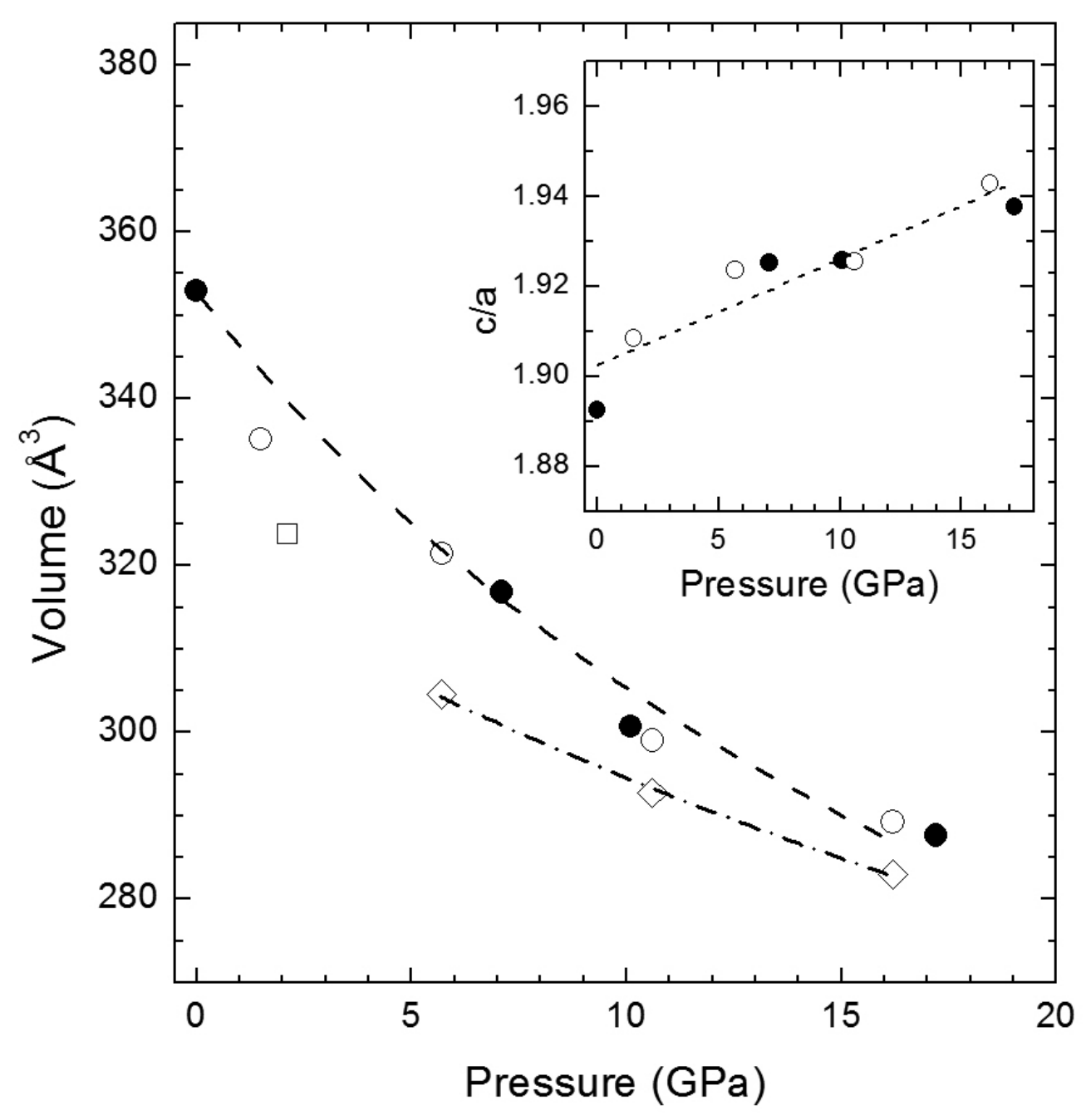

